Rike Balzuweit* und Martin Nissen

\title{
An der Zukunft bauen: Die Norderweiterung der Universitätsbibliothek Heidelberg und ihre aktuellen Innovationsfelder
}

DOI 10.1515/bfp-2016-0058

Zusammenfassung: Im Juli 2015 wurde der neue Lesesaal eröffnet und damit die Norderweiterung der Universitätsbibliothek Heidelberg vorerst abgeschlossen. Auf 6500 Quadratmetern entstand eine Lese- und Lernlandschaft mit rund 1000 Arbeitsplätzen, 15 Gruppenarbeitsräumen, 18 Carrels, 2 Schulungsräumen und 3 Loungebereichen. Der Beitrag bietet einen Überblick über die Baugeschichte, das Gebäudekonzept sowie das Dienstleistungsangebot und zeigt die Perspektiven in den Innovationsfeldern Integration des Bibliothekssystems, Erweiterung der E-Bibliothek und Ausbau der Publikationsdienste auf.

Schlagwörter: Universitätsbibliothek Heidelberg; Bibliotheksbau; Innovationsmanagement

\section{Building the Future: The Northern Extension of Heidelberg University Library and its Areas of Innovation}

\begin{abstract}
In July 2015, the new reading room was opened and the northern extension of Heidelberg University Library was completed for the moment. Covering 6,500 square meters, a reading, and learning landscape with about 1,000 working places, 15 group study rooms, 18 carrels, 2 classrooms, and 3 lounge areas were built. This article provides an overview of the architectural history, the construction concept, and the service portfolio and shows the perspectives for future developments, i.e. the integration of the library system, the expansion of the e-library, and the publication services.
\end{abstract}

Keywords: Heidelberg University Library; Library building; Innovation Management

\footnotetext{
*Kontaktperson: Rike Balzuweit, Balzuweit@ub.uni-heidelberg.de Dr. Martin Nissen, Nissen@ub.uni-heidelberg.de
}

Inhalt

1 Einleitung und Ausgangslage . . . . . . . . . . 452

2 Zur baulichen Situation . . . . . . . . . . . . . 454

3 Das Triplex-Gebäude bis zum Beginn der Sanierungsmaßnahmen . . . . . . . . . . . . . . 455

4 Bau- und Sanierungsphase . . . . . . . . . 458

5 Raumkonzept . . . . . . . . . . . . . . . . . . 459

6 Dienstleistungen................ 461

7 Innovationsfelder an der Heidelberger

Universitätsbibliothek . . . . . . . . . . . . . 462

8 Bauliche Erneuerung der Universitäts-

bibliothek ................ . . 463

9 Integration des Bibliothekssystems ... . . . 463

10 Ausbau der E-Bibliothek . . . . . . . . . . . . . . . 464

11 Publikationen, Forschungsdaten, Digitale

Editionen ... . . . . . . . . . . . . . . . . 464

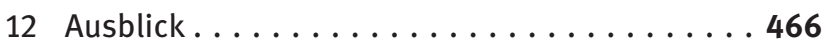

\section{Einleitung und Ausgangslage}

Die Gestaltung der Zukunft im Bewusstsein der eigenen Geschichte zählt zu den Leitgedanken der Universitätsbibliothek Heidelberg. Die älteste Universitätsbibliothek Deutschlands versteht sich als modernes Dienstleistungszentrum, das internationale Entwicklungen im Bibliothekswesen nicht nachvollzieht, sondern aktiv mitgestaltet. Dies betrifft den Ausbau von Services für die 47300 aktiven Nutzer vor Ort ebenso wie die Entwicklung neuer Angebote für die Schwerpunktfächer Kunstgeschichte, Asien- sowie Altertumswissenschaften.

Die Attraktivität der Dienstleistungen spiegelt sich in den hohen Nutzungszahlen wider. Laut Deutscher Bibliotheksstatistik zählt die Universitätsbibliothek Heidelberg seit Jahren zu den am stärksten genutzten Universitätsbibliotheken in Deutschland. Mit 9 Millionen Downloads aus der E-Bibliothek, 1,5 Millionen Buchausleihen sowie 2,6 Millionen Besuchen an den Hauptstandorten belegt sie seit 2010 die Spitzenposition unter den zweischichtigen Universitätsbibliotheken im Bibliotheksindex 
BIX. ${ }^{1}$ Insgesamt werden 31000 Studierende, 7000 Doktoranden, 5600 Wissenschaftler aus zwölf Fakultäten und ein interessiertes Fachpublikum mit wissenschaftlicher Literatur in gedruckter und elektronischer Form versorgt. Das digitale Angebot lizenzierter Quellen umfasst 3200 Datenbanken, 106000 E-Journals und 550000 E-Books.

Dieser hohen Attraktivität der digitalen Angebote sowie der starken Nutzung der Bibliothek vor Ort stand bis zum Jahr 2015 eine angespannte räumliche Situation am Hauptstandort in der Altstadt gegenüber. Zwar verfügt die Universitätsbibliothek Heidelberg auch baulich über eine bis in die Mitte des 15. Jahrhunderts zurückreichende Tradition. 1905 wurde dann der von dem Karlsruher Architekten Josef Durm (1837-1919) nach der Konzeption des Heidelberger Oberbibliothekars Karl Zangemeister (18371902) entworfene Altbau bezogen, in dem sie bis heute residiert. Dieser Bau, insbesondere der Magazintrakt, stieß aufgrund der Expansion des Bestandes nach dem Zweiten Weltkrieg jedoch wiederholt an seine Grenzen. Verschiedene Erweiterungsmaßnahmen konnten diese Raumnot jeweils nur kurzfristig lindern. So wurden in den 1950erJahren im Südflügel des Altbaus Zwischendecken eingebaut, Mitte der 1970er-Jahre im nördlich angrenzenden Triplex-Gebäude erste Flächen genutzt sowie in den 1980erJahren im Südflügel weitere Zwischenebenen eingezogen, diesmal in Form von Galerien. Hinzu kamen seit den späten 1980er-Jahren der Bau des Tiefenmagazins sowie die Anmietung externer Flächen im Stadtgebiet als Ausweichmagazine.

In den 1990er-Jahren wurden die Platzprobleme so drängend, dass die Universitätsbibliothek einen Antrag auf Bezug weiterer Flächen im Triplex-Gebäude vorlegte. Dem entgegen beförderten der Einzug des Medienwandels und das Wachstum der digitalen Bibliothek in den späten 1990er-Jahren bei den Bauverantwortlichen Zweifel, ob Bibliotheken als Lernorte in Zukunft überhaupt noch genutzt werden würden. Sowohl hinsichtlich der Buchausleihen als auch der Bibliotheksbesuche wiesen die 2000erJahre dann jedoch in eine andere Richtung. 2006 erreichte das Tiefenmagazin seine Kapazitätsgrenze. 2010 wurde mit 1,9 Millionen Ausleihen der Spitzenwert in der Nutzung der Buchbibliothek erreicht. Im gleichen Jahr verzeichneten die Besucherzahlen mit 2,3 Millionen Besuchen an den Hauptstandorten einen Rekord, der trotz wachsender Bedarfe aufgrund räumlicher Beschränkung nicht weiter zu steigern war. 1100 Arbeitsplätze in der Hauptbibliothek einschließlich der Zweigstelle bei einer Zahl von 31000 Studierenden erwiesen sich als zu knapp bemessen.

1 Alle Zahlen mit Referenzjahr 2015 (Stand 31.12.2015).
Insbesondere die Hauptbibliothek in der Altstadt mit ihren 600 Arbeitsplätzen war deutlich überlastet, so dass ein Teil der Studierenden auf die größeren Bereichs- und Institutsbibliotheken auswich.

Die Nutzungssituation in der Hauptbibliothek stellte sich zudem improvisiert dar. Der Lesesaal als zentraler Lern- und Arbeitsort war auf die beiden oberen Geschosse des historischen Magazintraktes verteilt, dessen niedrige Raumhöhen zumal bei Vollbelegung einen beengten Eindruck vermittelten.

Der Antrag aus den 1990er-Jahren auf Erweiterung der Flächen, der 2004 erneuert wurde, bewegte sich in einem hochkompetitiven Umfeld. In der Heidelberger Altstadt stoßen viele historische Bauten, in denen Institute und Seminare untergebracht sind, regelmäßig an ihre Grenzen. Neubauflächen sind rar. Die allgemeine Raumnot wurde durch den Erfolg der Universität Heidelberg in der Exzellenzinitiative 2007 und das starke Wachstum der Universität in den Folgejahren noch verschärft.

Die Bewilligung der Sanierung und der Bezug der dringend benötigten zusätzlichen Flächen in dem nördlich an den Altbau anschließenden Triplex-Gebäude wurden erst durch zwei Entwicklungen möglich: Den 2009 erfolgten Auszug der dort untergebrachten sozial- und wirtschaftswissenschaftlichen Institute sowie die Aufteilung der umfangreichen Baumaßnahme in zwei Bauabschnitte, die sich über insgesamt sechs Jahre hinzogen.

Durch den Abschluss der Sanierung des Triplex-Gebäudes im Sommer 2015 konnte die Servicequalität der Universitätsbibliothek Heidelberg grundlegend verbessert werden. Rund 1000 Arbeitsplätze auf 6500 Quadratmetern Nutzfläche sowie zusätzliche Stellflächen für rund 200000 Bände bedeuten nicht nur eine quantitativ und qualitativ langfristige Verbesserung für die Hauptbibliothek in der Altstadt, sondern entspannen die Nutzungssituation im gesamten Heidelberger Bibliothekssystem. Die feierliche Übergabe an die Universität fand am 29. Juli statt. Für die Baumaßnahme stellte das Land Baden-Württemberg 20 Millionen Euro zur Verfügung. Seit Eröffnung wird allein der Lesesaal von durchschnittlich rund 2300 Nutzern pro Tag besucht. In Bezug auf das Vorjahr bedeutet dies eine Steigerung um $88 \%$.

Der folgende Beitrag bietet einen Überblick über Geschichte, Verlauf und Abschluss der Baumaßnahme. Zugleich öffnet er den Blick von der Benutzung vor Ort auf weitere Schwerpunkte der zukünftigen Bibliotheksarbeit. Es ist das Ziel der Universitätsbibliothek Heidelberg, die bestmögliche Unterstützung für Forschung und Lehre an der Universität Heidelberg und darüber hinaus zu bieten. Dafür stellt der neue Lesesaal einen wichtigen Meilenstein dar. 


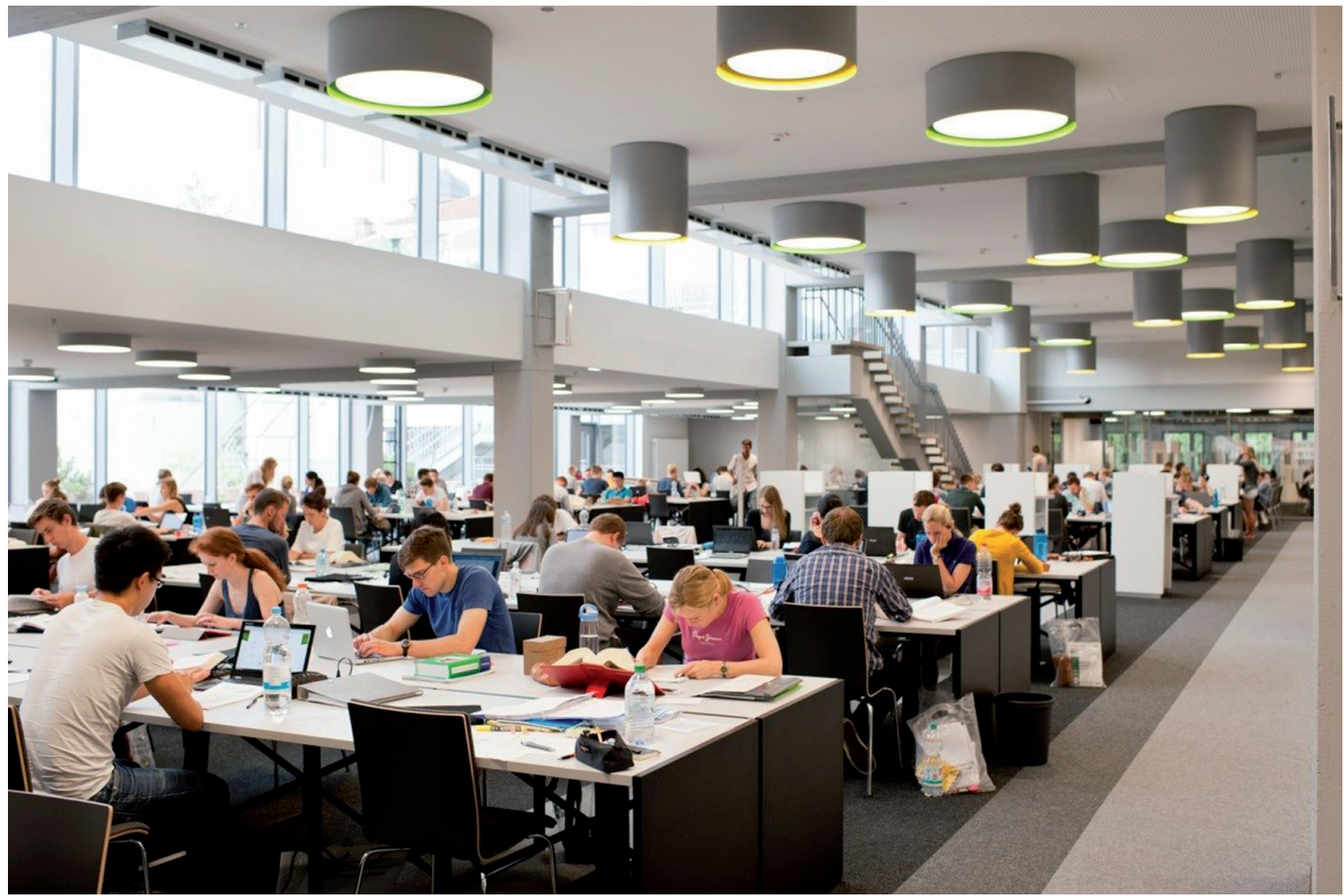

Abb. 1: Triplex-Lesesaal: Hauptnutzungsflächen im 2. OG. Foto: Michael Miethe

\section{Zur baulichen Situation}

Die Universitätsbibliothek Heidelberg umfasst baulich die Vierflügelanlage des historischen Altbaus von 1905 mit dem repräsentativen Südflügel und den drei Magazinflügeln rund um den Innenhof. Der Altbau stößt im Norden an das von Lothar Götz entworfene Triplex-Gebäude aus den 1970er-Jahren, dessen Mitteltrakt nach Norden terrassiert abfällt und in zwei Flügeln ausläuft, die einen weiteren Innenhof umschließen. Der Durm'sche Altbau beherbergt im Südflügel das Informationszentrum mit der zentralen Erstauskunft, das Ausleihzentrum, das Multimediazentrum mit dem PC-Pool, den Handschriftenlesesaal, die Ausstellungsräume sowie die Direktion und einen Teil der Verwaltung. In den drei Flügeln des Magazintraktes werden auf fünf Geschossen die Erwerbungsjahrgänge 1981-2016 in NC-Aufstellung zur sofortigen Ausleihe angeboten. Der Nordflügel des Magazintraktes dient als Übergang zwischen dem vierten Obergeschoss des Altbaus und dem neuen Lesesaal im Triplex-Gebäude. Hier ist auch die Zugangskontrolle für den Lesesaal sowie die zentrale Lesesaaltheke mit einem Lesebereich für besonders schützenswerte Bestände angesiedelt.
Alt- und Neubau bilden - vor und nach Sanierung des Triplex-Gebäudes - hinsichtlich der Farbgebung, der Formensprache und der Lichtverhältnisse einen starken Kontrast, der augenfällig ist. Der späthistoristische, im Geist der französischen und deutschen Renaissance gestaltete Altbau weist florale Muster auf, fließende gerundete Formen, dezente Naturtöne und eine Materialvielfalt aus Sandstein, gelbem Jura, beige-rotem Terrazzo-Boden sowie teils naturbelassenen, teils dunkel gebeizten Eichenverkleidungen. Die Wände und Säulen sind vielfach farbig lasiert oder auch marmoriert angelegt. Bei den Ornamenten überwiegen Gold, Blau und rote Sandsteintöne. Für Brüstungen und Sockelelemente wurde verschiedenfarbiger Marmor eingesetzt, dazu schmiedeeiserne Geländer. Tür- und Fenstergitter sind teilweise verziert und mit Rankenwerk versehen. Der erhabene Gesamteindruck wird durch die lichten Raumhöhen sowie die Ikonografie der Schmuckelemente verstärkt.

Der Magazintrakt ist in seiner funktionalen Ausrichtung deutlich nüchterner gehalten als der schlossartige Südflügel und mildert so den architektonischen Bruch zwischen Alt- und Neubau. Die Stahlstützen, die Unterzüge und die Rippen der Tonnengewölbedecke sind grau 


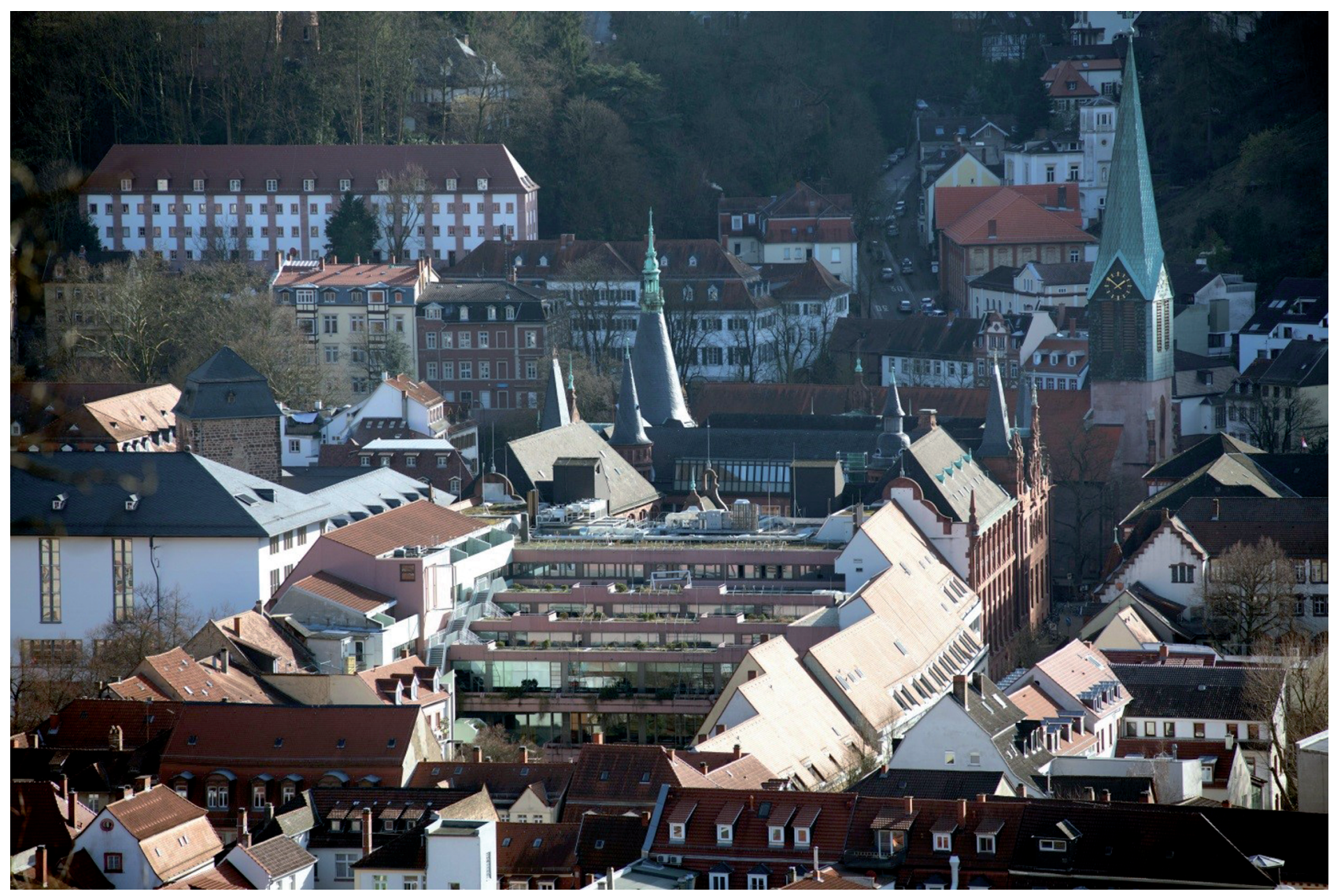

Abb. 2: Triplex-Gebäude und historischer Altbau der Universitätsbibliothek Heidelberg von der nördlichen Neckarseite aus aufgenommen. Foto: Uwe Bellm

gestrichen, die Wandflächen weiß verputzt. Die durch Zwischenpfeiler verstärkte Stahlkonstruktion über fünf Geschosse vermittelt den Eindruck einer streng gegliederten, ästhetisch ansprechenden Funktionseinheit. Die niedrigen Geschosshöhen von maximal 2,32 Metern kontrastieren deutlich zu der Doppelgeschossigkeit des Südflügels und betonen den Unterschied zwischen der repräsentativen Großzügigkeit des Südflügels gegenüber der funktionalen Strenge der Büchermagazine.

Das Triplex-Gebäude aus den 1970er-Jahren hieß offiziell „Seminargebäude Grabengasse/Sandgasse“. Der Name Triplex stammt aus dem letztlich erfolgreichen Entwurf der Architekten Götz, Heuser, Unruh aus der Wettbewerbsphase von 1969/70 und bezieht sich auf die dreifache Nutzung als Mensa, Instituts- und Bibliotheksgebäude. Der Bau schließt unmittelbar nördlich an den Magazintrakt an und bildet zusammen mit dem Altbau entlang der Grabengasse eine Flucht von 120 Metern. Das Gebäude setzt hinsichtlich der Formensprache, der verwendeten Materialien sowie der Farbgebung einen Kontrapunkt zu dem Durm'schen Prachtbau einerseits sowie zu der Kleinteiligkeit der nördlich anschließenden Bürgerhäuser mit ihren Sprossenfenstern und den für die Heidelberger Altstadt charakteris- tischen unterschiedlichen Dachhöhen andererseits. Dieser Kontrast wurde bei der grundlegenden Sanierung in den zwei Bauphasen zwischen 2009 und 2015 bewusst beibehalten, um die individuelle Baugeschichte beider Gebäude auch weiterhin zu zeigen und nicht zu verdecken.

\section{Das Triplex-Gebäude bis zum Beginn der Sanierungsmaßnahmen}

In den 1950er-Jahren beschloss die Universität Heidelberg, die Sozial- und Geisteswissenschaften in der Altstadt zu belassen, die Naturwissenschaften sowie die Medizin dagegen im bis dahin weitgehend unbebauten Neuenheimer Feld auf der nordwestlichen Neckarseite zu konzentrieren. Dies führte dazu, dass bisherige Klinikgebäude im Westen der Altstadt sowie im westlich angrenzenden Stadtteil Bergheim frei wurden und von geistes- und sozialwissenschaftlichen Instituten nachbelegt werden konnten. Der Raumdruck blieb aufgrund der raschen Expansion der Universität in den 1960er-Jahren indessen groß. Als Standort 

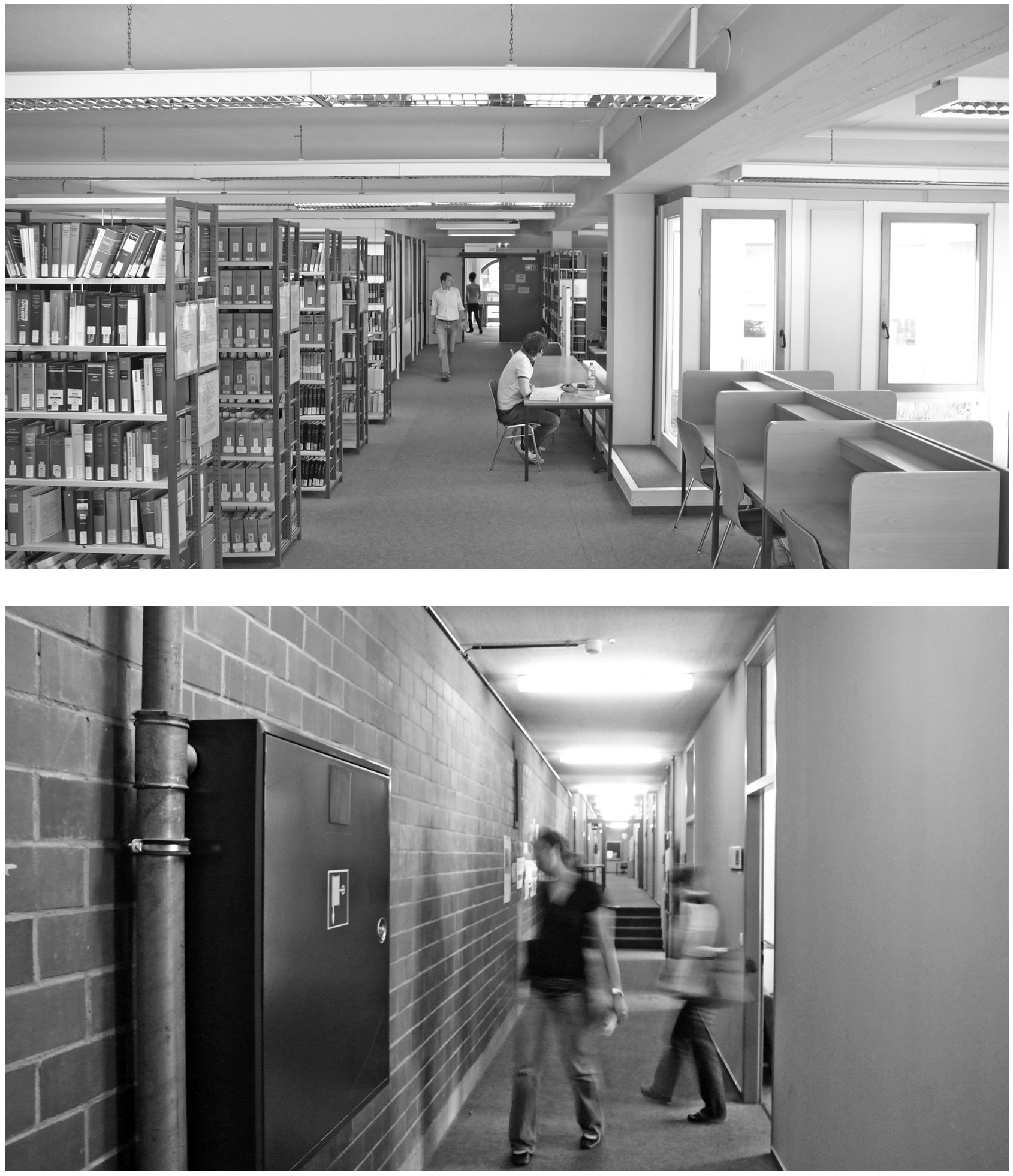

Abb. 3 a und 3b: Das Triplex-Gebäude vor der Sanierung. Fotos: Uwe Bellm

für ein neues Seminar- und Institutsgebäude wurde das Areal am Universitätsplatz nördlich der Universitätsbibliothek zwischen Sand- und Grabengasse ausgewählt. 1969 führte die Neue Heimat Städtebau einen nicht öffentlichen städtebaulichen Wettbewerb zur Unterbringung der Mensa sowie der wirtschaftswissenschaftlichen Institute durch. ${ }^{2}$ Aufgrund der - wie es in der Begründung der Gutachter-

2 Vgl. im Folgenden Kranz $(1985,204)$. 
Kommission hieß - großzügigen Freiflächen sowie der „großräumige[n] Lösung im Innern des Komplexes“ fiel die Entscheidung 1970 zugunsten des Entwurfs „Triplex“ der Architekten Götz, Heuser, Unruh. ${ }^{3}$ In der Stadt und an der Universität formierte sich Widerstand gegen die Bauplanungen. Die Gegner sprachen sich gegen eine Zerstörung der typisch kleinräumigen Altstadtarchitektur aus und plädierten von einem denkmalpflegerischen Standpunkt aus für eine Erhaltung der bisherigen Bebauung. So erfolgte die Baufreigabe erst im Oktober 1974, an die sich der Abriss von 10 Wohnhäusern in der Sand- und Grabengasse anschloss. Der Raumbedarfsplan musste mehrfach überarbeitet werden und wurde in seiner endgültigen Fassung erst 1976 beschlossen. Insbesondere die Farbgebung des Außenanstrichs blieb ein Heidelberger Politikum. Sie wurde in mehreren Probeanstrichen, die sich in die Umgebung einpassen sollten, vorgestellt und erst 1978 mit einer Abstufung von Grün-, Gelb-, Braun- und Grautönen genehmigt.

Trotz dieser Verzögerungen erfolgte die Fertigstellung des Gebäudes bereits 1978. Mit rund 11000 Quadratmetern stellt das Triplex-Gebäude seitdem die größte zusammenhängende Nutzfläche in der Heidelberger Altstadt dar. Der Westtrakt des Gebäudes wurde aus Denkmalschutzgründen aufgemauert, die übrigen Trakte wurden als Stahlbetonkonstruktion mit Fertigteilen hergestellt, die vor die Fassade gehängt sind. Der Außenbereich war mehrfarbig, innen überwog Sichtbeton mit weiß gestrichenen Wänden. Die Trennung zwischen Mitteltrakt und den Seitenspangen war als offenes Ziegelmauerwerk ausgeführt. Räumlich wurde das Gebäude in den Seitentrakten in kleinteilige Büroräume und innen liegende lange fensterlose Erschließungsgänge gegliedert. Der Mitteltrakt ist durch große Freiflächen und eine große geschossübergreifende Abgangsspindel zwischen erstem Obergeschoss und Erdgeschoss gekennzeichnet. Dem nach Norden abfallenden Terrain passt sich die Ostspange durch Geschossversatz mit einigen Treppenstufen an. Zur Innenhofseite nach Norden hin bringen große Verglasungen sowie stufenförmig angesiedelte Außenterrassen viel Licht in den Gebäudekörper.

In Anpassung an die städtebauliche Enge der Heidelberger Altstadt bot das Triplex-Gebäude bis zur Generalsanierung eine Vielzahl unterschiedlicher Nutzungsformen. In den beiden Untergeschossen entstand eine der ersten Tiefgaragen in der Heidelberger Innenstadt, für die das Gebäude auf der Westseite großflächig geöffnet wurde. Das Erdgeschoss gliedert sich in die gepflasterte Haupt-

3 Altstadterneuerung (1970). halle mit der Cafeteria, den Aufgängen zur Mensa mit 660 Plätzen im ersten Obergeschoss sowie in die erwähnte große Abgangsspindel. In den oberen Geschossen zwei bis vier wurden in Nord-Südausrichtung entlang der Seitengassen das Institut für Sozial- und Wirtschaftsgeschichte, das Institut für International Vergleichende Wirtschaftsund Sozialstatistik, das Alfred-Weber-Institut für Sozialund Staatswissenschaften, das Institut für Soziologie und die Dekanate der Fakultät für Sozial- und Verhaltenswissenschaften, der Neuphilologischen und der Wirtschaftswissenschaftlichen Fakultät untergebracht. Im Mitteltrakt war im zweiten Stock die Fachbibliothek des Alfred-Weber-Instituts zusammen mit der Bibliothek des Instituts für Wirtschafts- und Sozialgeschichte aufgestellt, im dritten Stock darüber - und durch einen Luftraum verbunden die Bibliothek des Instituts für Soziologie. Im westlichen Seitenflügel zur Sandgasse hin befanden sich auf den unteren Ebenen weitere Einrichtungen: im zweiten Geschoss das Institut für Ethnologie, dessen Bibliotheksbestände zwischenzeitlich mit der Bibliothek des Instituts für Soziologie zur gemeinsamen Bibliothek der Institute für Soziologie und Ethnologie verschmolzen waren, im Erdgeschoss und im Zwischengeschoss das Institut für Sprachen und Kulturen des Vorderen Orients mit den Abteilungen Islamwissenschaft und Iranistik samt Institutsbibliothek. Die Universitätsbibliothek erhielt die Möglichkeit, in den beiden oberen Geschossen Durchbrüche zwischen dem historischen Magazintrakt und dem Triplex-Gebäude herzustellen, um Teile des Triplex als Lesesaal für die Rechts-, Sozial- und Wirtschaftswissenschaften sowie als Zeitschriftenmagazin zu nutzen. Diese Nutzung umfasste jedoch lediglich Geschossflächen in einer Größenordnung von rund 300 Quadratmetern. Nach Bezug des Gebäudes wurden Teile der Seitenspangen zu den Gassenseiten hin gewerblich genutzt. Nebennutzer waren ein Postamt und eine Buchhandlung. Ergänzend wurden Wohnungen sowie weitere Verwaltungsräume eingerichtet.

Die Bewertung des Triplex-Gebäudes aus den 1970erJahren muss aus der damaligen Entstehungssituation heraus vorgenommen werden. Die zahlreichen Nutzungsanforderungen verschiedener Institute waren nur durch eine im Vergleich zu den massiven zusammenhängenden Außenflächen kleinteilige innere Erschließung zu realisieren. Die Umsetzung führte zwar zu einer hohen Raumnutzung, war jedoch nur durch lange innenliegende ErschlieBungszonen erreichbar, die die Orientierung erschwerten und einen insgesamt dunklen und engen Raumeindruck vermittelten. Das Gebäude wies 2009 nach jahrzehntelanger Nutzung erhebliche bautechnische Mängel auf, die sich u. a. in Undichtigkeiten im Bereich der Außenterrassen äußerten. Die Sanierung und Neugestaltung dieses für Heidel- 
berger Verhältnisse umfangreichen Gebäudekomplexes war somit überfällig.

Im Juni 2004 formulierte die Universitätsbibliothek eine erste Nutzungsanforderung zur Ausdehnung der Bibliothek in das Triplex-Gebäude hinein. Während andere Universitätsbibliotheken des Landes - so Ulm, Mannheim, Tübingen, Karlsruhe und Konstanz - in den frühen 2000er-Jahren zum Teil beträchtliche Ausbauten mit erheblich größeren Nutzungsflächen erhielten, war das Raumangebot der Universitätsbibliothek Heidelberg zu diesem Zeitpunkt quantitativ wie qualitativ auf dem Stand der späten 1980er-Jahre. Insbesondere bei der Zahl der Arbeitsplätze fiel Heidelberg deutlich hinter vergleichbare Bibliotheken zurück. So gab es 2004 in der Haupt- und Zweigbibliothek zusammen nur rund 660 Lese- und EDVArbeitsplätze. Eklatant war der Mangel auch bei der Zahl der Gruppenarbeitsplätze. Die UB Heidelberg verfügte über lediglich vier Gruppenarbeitsräume zu je zehn Quadratmetern. Aufgrund der Raumnot in der Hauptbibliothek gab es keine Möglichkeit, das Dienstleistungsangebot auszuweiten und etwa spezielle Arbeitsbereiche für Examenskandidaten oder Doktoranden einzurichten. Hinzu kam bei einem jährlichen Zugang von rund 40000 Medieneinheiten die Überlastung des Tiefenmagazins, das sich 2004 kurz vor der Erschöpfung der Kapazitätsgrenzen befand.

Voraussetzung für den Beginn der dringend erforderlichen Sanierungsmaßnahmen im Triplex-Gebäude war der Auszug von Instituten der Sozial- und Wirtschaftswissenschaften, der wiederum erst durch den Umzug der Ludolf-Krehl-Klinik aus dem Stadtteil Bergheim ins Neuenheimer Feld möglich wurde. 2009 war es dann soweit. In das freie Klinikgebäude zogen die Institute der Wirtschaftsund Sozialwissenschaften. Die Universitätsbibliothek führte die Bibliotheken der Fakultät für Wirtschafts- und Sozialwissenschaften mit der zuvor anderweitig untergebrachten Bibliothek des Instituts für Politische Wissenschaft zur Campus-Bibliothek Bergheim (CBB) zusammen. Das Triplex-Gebäude wurde dadurch freigestellt und stand für eine Nachnutzung durch die Universitätsbibliothek zur Verfügung.

\section{Bau- und Sanierungsphase}

Die Sanierung des Triplex-Gebäudes in den Jahren 20092015 übernahm das Heidelberger Architekturbüro ap88 unter Leitung von Uwe Bellm. Beteiligte Akteure waren neben den Architekten das Universitätsbauamt Heidelberg, das Dezernat Planung, Bau und Sicherheit der Universität sowie die Verantwortlichen der Universitätsbibliothek.
Mit der Sanierung waren vier bauliche Ziele verbunden: eine ausreichende Anzahl von rund 1000 neuen Arbeitsplätzen, die Realisierung eines zeitgemäßen Lichtkonzepts, eine ruhige Arbeitsatmosphäre sowie ein angenehmes Raumklima durch eine moderne Lüftungstechnik. Diese Ziele wurden erreicht und durch ein differenziertes Dienstleistungsangebot für verschiedene Nutzergruppen ergänzt.

Neben den bautechnischen Anforderungen, die sich aus der Sanierung eines Gebäudes aus den 1970er-Jahren ergaben, stellte die Verbindung von Alt- und Neubau eine zentrale Herausforderung für das Planungsbüro dar. Der Bruch zwischen den beiden Gebäudeteilen ergibt sich nicht nur durch die unterschiedlichen Baustile, sondern auch durch einen Versprung der Geschosshöhen, der den topographischen Abfall des Terrains nach Norden ausgleicht. So mussten vier Geschosse des Triplex-Gebäudes an fünf Geschosse des historischen Magazintraktes angeschlossen werden. Hinzu kam die Anforderung, die Planung so vorausschauend zu gestalten, dass sich auch das erste Geschoss des Triplex, in dem sich derzeit noch die Mensa des Studierendenwerks befindet, zu einem späteren Zeitpunkt an die Bibliothek angliedern ließe.

Das Pflichtenheft der Sanierung wurde - wie bei umfangreichen Baumaßnahmen im öffentlichen Bereich üblich - weithin durch den vorbeugenden Brandschutz geprägt. Das Brandschutzkonzept erarbeitete ein Fachgutachter in Zusammenarbeit mit dem örtlichen vorbeugenden Brandschutz der Feuerwehr. Besonders bemängelt wurde die unzureichende Erschließungssituation des großflächigen Gebäudes mit in Anzahl und Größe unterdimensionierten Treppenhäusern. Erforderlich zur Entflüchtung war daher die Neukonzeption eines Haupttreppenhauses zwischen Alt- und Neubau, zudem die Verbindung der nördlichen Außenterrassen zu einem zusätzlichen Fluchtweg ins Freie. Aufgrund der großen Gebäudetiefe wurde auch innerhalb des Lesesaals eine weitere Treppe zwischen dem zweiten und dritten Stockwerk hergestellt.

Der erste Bauabschnitt verlief von 2009 bis 2012 und umfasste die komplette Außeninstandsetzung inklusive Betonsanierung, die Entkernung des Gebäudes sowie die Beseitigung der Schadstoffe. Für die Entkernung wurde ein Großteil der Innenwände einschließlich des offenen Ziegelmauerwerks entfernt und durch Glaswände ersetzt. Hierdurch wird der Blick auf die Seitenflügel sowie die Seitengassen frei. Nach West und Ost entstanden Sichtachsen, die eine wesentlich bessere Orientierung innerhalb des Gebäudes ermöglichen.

Im Zentrum des ersten Bauabschnitts stand die Sanierung des Ostflügels. Neben öffentlichen Bereichen wurden in der Ostspange auf dem zweiten bis fünften Stockwerk 


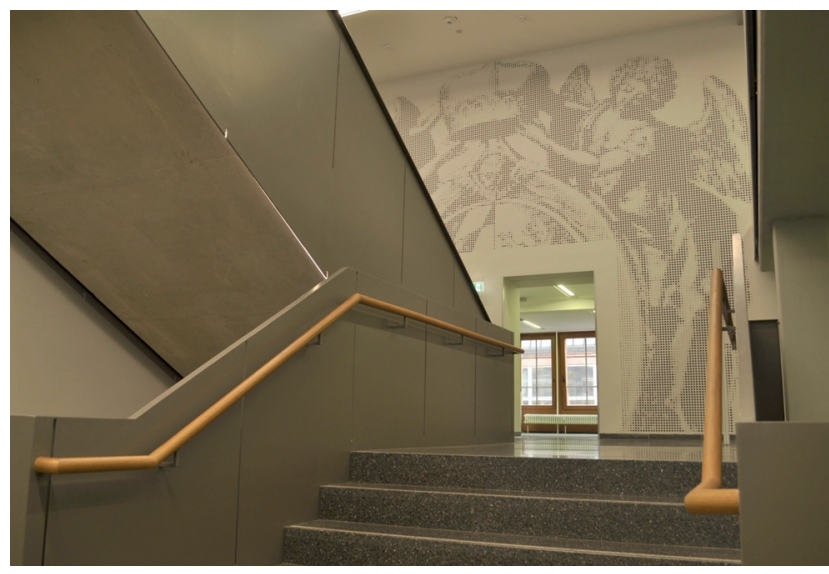

Abb. 4: Das zentrale Treppenhaus im Triplex-Gebäude. Foto: Martin Nissen

interne Verwaltungsflächen mit zusammen 700 Quadratmetern für die medienbearbeitenden Abteilungen sowie für Fachreferenten und Projektmitarbeiter geschaffen. Im September 2012 wurden die ersten Nutzerflächen in Betrieb genommen. Diese bieten auf den Stockwerken zwei und drei rund 100 Arbeitsplätze, acht Gruppenarbeitsräume, die Präsenzbestände der Rechts-, Wirtschafts- und Sozialwissenschaften sowie zwei große Sanitärbereiche. Parallel zur Inbetriebnahme der erweiterten Flächen verlängerte die Bibliothek die täglichen Öffnungszeiten um drei Stunden von 22 auf 1 Uhr nachts unter Einsatz eines Wachdienstes.

Der zweite Bauabschnitt von 2013 bis 2015 war wesentlich umfangreicher. Er umfasste die komplette Sanierung und technische Erneuerung der Hauptflächen im Mitteltrakt auf den drei oberen Stockwerken des Gebäudes sowie die Instandsetzung der Westspange. Wie erwähnt, war hierfür der Einbau eines neuen zentralen Treppenhauses erforderlich, das zum ersten Mal den historischen Altbau mit dem Triplex-Gebäude in strukturierter Weise verbindet. Davor hatte es nur auf den zwei oberen Altbau-Magazingeschossen je einen improvisierten Durchstich gegeben. Das neue zentrale Treppenhaus greift die unterschiedlichen Geschosshöhen auf, indem es diese durch versetzte Treppenabsätze als raumstrukturierendes Element nutzt. Die eingesetzten Materialien mit einem Betonwerkstein als Fußbodenbelag sowie einem verschweißten und lackierten Treppengeländer aus Schwarzstahl mit einem Handlauf in Eiche Natur vermitteln einen repräsentativen Raumeindruck. Konzeptionell stellt der Zugangsbereich zum neuen Lesesaal - analog zum Eingangsbereich zum historischen Altbau - einen Übergang zu den nördlich anschließenden Funktionsbereichen her.

Ein Aufzug im zentralen Treppenhaus ermöglicht den barrierefreien Übergang zwischen den Stockwerken. Der
Zugang zu den eigentlichen Leseflächen erfolgt dann aus dem Treppenhaus durch von Bewegungsmeldern gesteuerte Glasschiebetüren, die akustisch den lauteren Bereich auf den Hauptbewegungsflächen im Treppenhaus von den ruhigeren Arbeitsbereichen abtrennen und zudem als Barriere für den Luftaustausch über eine Luftwechselanlage dienen.

\section{Raumkonzept}

Die Gestaltung der Flächen auf den drei Stockwerken des neuen Triplex-Lesesaals folgt dem Leitgedanken der Einheit von Bau und Funktion. „Des Körpers Form sei seines Wesens Spiegel“4 - bereits für den historischen Altbau von 1905 hatte der leitende Baumeister Josef Durm dieses Zusammenspiel angeführt, um die bauliche Gliederung wie die unterschiedliche Gestaltung von schlossähnlichem Repräsentations- und funktionalem Magazintrakt zu begründen: „Man hat im Verlaufe der letzten Jahre einsehen gelernt, dass es bei öffentlichen Büchereien weniger darauf ankommt, einen gleichmäßig entworfenen palastähnlichen Bau an die Straße zu stellen, als vielmehr darauf, aus der Eigenart des Bedürfnisses den Bau herauszuarbeiten und sein Äußeres danach zu gestalten. ${ }^{\text {"5 }}$

Die Flächen auf den beiden großen unteren Stockwerken im neuen Lesesaal unterteilen sich in die Hauptflächen im Mitteltrakt und die Flügel auf der Ost- und Westseite des Gebäudes. Die Unterteilung erfolgt durch Glaswände mit elektronisch gesteuerten Durchgangstüren, ebenfalls aus Glas, die eine akustische und gestalterische Abtrennung herbeiführen und gleichzeitig den Blick auf die Grabenund Sandgasse ermöglichen. In den Seitenflügeln überwiegen Sitzarbeitsplätze, die in Gruppen zusammengefügt sind und dem ruhigen und konzentrierten wissenschaftlichen Arbeiten dienen. Diese Arbeitsplätze zeichnen sich durch eine besonders hochwertige Ausstattung mit Einzelleuchten, Trennwände zwischen den Tischseiten sowie Steckdosen an jedem Arbeitsplatz aus. Das Farbkonzept in den Seitenspangen signalisiert mit einer fast monochromen, schwarz-weißen Farbgebung Innerlichkeit und eine Reduzierung auf das stille wissenschaftliche Arbeiten. Hier steht der einzelne Nutzer im Vordergrund. Wesentliche Merkmale sind Ruhe, Konzentration, Zurückgezogenheit und Kleinräumigkeit. Nochmals vertieft wird dieses Konzept in den Einzelarbeitskabinen (Carrels) im Ostflügel so-

4 Zit. nach Grammbitter-Ostermann $(1985,198)$.

5 Grammbitter-Ostermann $(1985,197)$. 
wie in der laptopfreien Zone im nördlichen Teil des Westflügels.

Bei den Hauptnutzungsflächen im Mitteltrakt handelt es sich um eine Mischzone. Hier folgen von Süden nach Norden bzw. vom Eingang zur Fensterfront hin Präsenzbestände, Auskunftsbereich, Zonen bequemen Lesens und wissenschaftliche Arbeitsplätze in großflächig gestalteten Raumabschnitten aufeinander. Geprägt werden die Flächen von ausgedehnten Regalreihen der Präsenzbibliothek einerseits und großen Gruppen wissenschaftlicher Arbeitsplätze andererseits. Wesentliche Merkmale sind Großzügigkeit und Offenheit, die durch die Doppelgeschossigkeit im Bereich der Fensterfront im zweiten Obergeschoss noch betont wird. Der Luftraum öffnet sich hier zum dritten Obergeschoss, das - von der Fensterfront aus betrachtet - wie eine Galerie ins zweite Obergeschoss eingehängt ist. Die funktionale Differenzierung zwischen den Seitenflügeln und den Hauptflächen spiegelt sich auch in der Wahl des Bodenbelags wider. Während die Hauptflächen durch breite Nadelfilzstreifen in drei unterschiedlichen Grautönen gestaltet sind, die durch wenige gelbe und grüne Farbinseln im Bereich der Informationstheke sowie der Loungebereiche kontrastiert werden, ist der Teppichboden in den Seitenspangen einheitlich anthrazitfarben. Auf den Hauptflächen entsteht ein bewegter Farbeindruck durch die Farbigkeit der Buchrücken, die sich von den weiß-schwarz und grau gestalteten Ausstattungselementen abheben.

In dieses Konzept fügt sich auch die Farbauswahl des Mobiliars ein, das ebenfalls durchgängig in schwarzen, weißen und grauen Tönen gehalten ist. Die Arbeitsflächen von Tischen und Ablagen sind dabei hellgrau ausgeführt. Einschnitte und Aussparungen wurden in einem dunkleren Basaltgrau ausgebildet. Die Ausführung der Korpusoberflächen erfolgte in Weiß. Funktional und gestalterisch bildet der neue Lesesaal eine bewusst sich selbst zurücknehmende Raumeinheit, die durch Bücher und Benutzer Farbigkeit und Bewegung erhält.

Mit Ausnahme des Standardregalsystems, der Sessel und Stühle wurde das Mobiliar vom Architektenteam entwickelt und von einer Schreinerei angefertigt. Das Tischsystem ist modular aufgebaut, ein Tisch besteht je aus einer Tisch- und zwei Seitenhartfaserplatten, die durch ein Metallgestänge in Kreuzverbindung Stabilität erhalten. Beliebig viele Tische können aneinander montiert werden. Diese Tischarbeitsplätze werden ergänzt durch sogenannte „Busse“, bei denen - seitlich an der Brüstung von Treppenabgängen - mehrere Arbeitsplätze hintereinander als Tisch-Stuhl-Einheit so gefertigt sind, dass die Arbeitsplatte mit einer kleinen Wandplatte abschließt, die dem vorne anschließenden Arbeitsplatz als Rückenlehne dient.
Die Hauptwege vom zentralen Treppenhaus zu den wissenschaftlichen Arbeitsplätzen werden beidseits von langen weißen Highboards flankiert, bei denen Steh- und Sitzarbeitsplätze abwechseln. Die Loungebereiche sind mit schlanken Hochlehnersesseln in einem Aubergineton ausgestattet. Weiße, fest auf dem Boden installierte Holzwürfel bieten Stromsteckdosen und Platz zur Ablage von Utensilien. Klassische Stehpulte runden das Mobiliar ab.

Eines der Ziele des Sanierungsvorhabens bestand ferner darin, den dunklen gedrückten Raumeindruck des ehemaligen Seminargebäudes aufzuheben und eine helle Ausleuchtung der Flächen herzustellen. Angesichts der Raumtiefe stellte dies eine zentrale Herausforderung dar, die die Einschaltung eines Lichtplaners erforderte. Konzeptionell sieht die Raumaufteilung vor, dass die Buchbestände im dunkleren Zugangsbereich aufgestellt sind. Die Arbeitsplätze befinden sich in den mit Tageslicht versorgten Seitenspangen und im Norden des Mitteltraktes, der sich mit einer großen Fensterfront zu den Terrassen öffnet und im vierten Geschoss den Blick auf den Heidelberger Philosophenweg freigibt. Die Beleuchtung erfolgt flächig von oben mit Aufbauleuchten unterschiedlichen Durchmessers, die teilweise als Tonnen abgependelt sind. Die Außenhaut dieser Tonnen-Leuchten ist hellgrau. Die Innenflächen sind gelb und grün gehalten, wobei von unten nur eine dezente Farbfuge zwischen Leuchte und Tonne von rund fünf Zentimetern sichtbar ist. Die Farbwahl ist wie bei den grüngelben Farbakzenten des Bodens im Lounge- und Thekenbereich eine Reminiszenz an die Erbauungszeit des Gebäudes, die 1970er-Jahre. Die variabel einsetzbaren Tonnen-Leuchten sind von entsprechend großer Höhe in den Doppelgeschossen und niedriger Höhe in den Zugangsbereichen und Seitenspangen. Auch lichttechnisch wird dabei der Leitgedanke der differenzierten Zonierung aufgegriffen. Auf den Hauptflächen im Mitteltrakt erfolgt die Lichtführung großflächig und breitstreuend, wohingegen in den Seitenspangen die Tonnen-Leuchten durch individuell einsetzbare Einzelplatzleuchten ergänzt werden.

Neben der Beleuchtung war die Erzeugung eines angenehmen Raumklimas ein weiteres Ziel der Sanierung. Zum Einsatz kommt eine Luftwechselanlage mit einem Zuluftvolumenstrom von 57000 Kubikmetern pro Stunde und einer Kälteversorgung zur Spitzenkühlung für die Hauptflächen im zweiten und dritten Geschoss. Die Benutzungsbereiche im Ostflügel, die bereits im September 2012 in Betrieb gingen, werden über eine weitere, kleiner dimensionierte Luftwechselanlage belüftet, die ebenfalls mit einer Vorkühlung für die heißen Sommermonate ausgestattet ist.

Die Kunst am Bau stammt von der Heidelberger Künstlerin Nicola Falley. Aus poliertem Edelstahl-Quadratrohr 


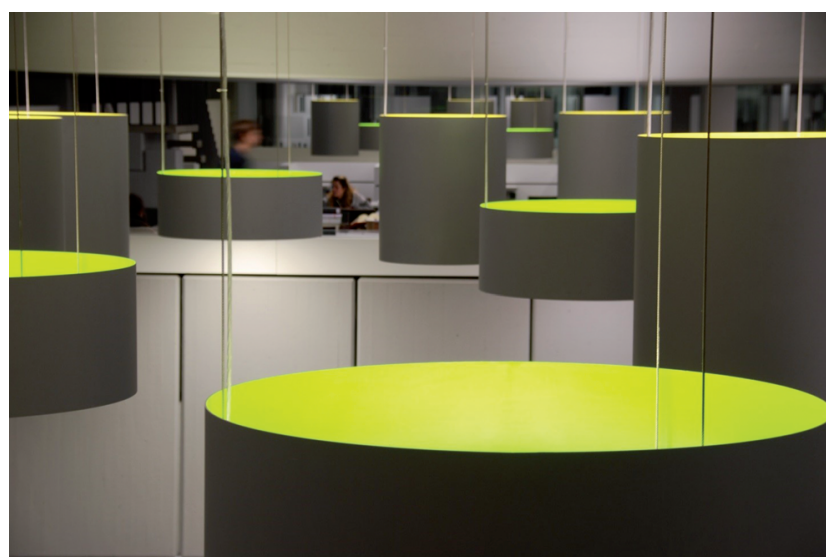

Abb. 5: Tonnen-Leuchten im Triplex-Lesesaal. Foto: Martin Nissen

hat sie in verschiedenen Größen stilisierte Bücher hergestellt, die an Säulen, auf einer Terrasse sowie an einer Außenwand montiert sind. In schlichter Zeitlosigkeit bildet ihr Kunstwerk das Wesen einer Bibliothek ab und fügt sich harmonisch in die zurückhaltende Raumgestaltung.

\section{Dienstleistungen}

Das Dienstleistungsangebot, das im Zuge der Sanierungsmaßnahme deutlich ausgeweitet wurde, wird an den Informationstheken gebündelt. Die Haupttheke im Zugangsbereich, der im vierten Geschoss des historischen Magazintraktes liegt, ist täglich von 8:30 bis $22 \mathrm{Uhr}$, am Wochenende von 9 bis $22 \mathrm{Uhr}$ besetzt. In Kernzeiten unter der Woche sowie am Wochenende von 9 bis $22 \mathrm{Uhr}$ wird die Haupttheke durch eine weitere Informationstheke im zweiten Geschoss des Triplex-Lesesaals ergänzt. Voraussetzung für den Ausbau des Dienstleistungsangebots ist ein qualifiziertes Auskunftsteam, das für den Betrieb in den Abendstunden sowie am Wochenende durch gut eingearbeitete und stetig fortgebildete studentische Hilfskräfte erweitert wird. Ab 22 Uhr erfolgt bis zur Schließung um 1 Uhr die Beaufsichtigung durch einen Wachdienst. Angesichts der hohen Nutzerzahlen und der Weitläufigkeit der zu betreuenden Bereiche ist der Personaleinsatz mit maximal vier Einsatzkräften für Jacken- und Taschenkontrolle, Ausgabe von schützenswerten Beständen, Lesesaalbestellungen und gebundenen Zeitschriftenbänden, die Information und Beratung an zwei Theken, die Bestandspflege sowie die Betreuung der Kopiergeräte und der elektronischen Angebote in den Gruppenarbeitsräumen effektiv gestaltet.

Die Buchbestände sind auf allen drei Stockwerken im jeweils dunkleren Innenbereich aufgestellt. Für den Präsenzbestand sind 500 Stellmeter für bis zu 90000 Bände reserviert. Der Schwerpunkt liegt auf den Rechts-, Wirt- schafts-, Sozial- und Geisteswissenschaften. Für die Naturund Lebenswissenschaften, die fachlich in der Zweigstelle im Neuenheimer Feld angesiedelt sind, wird in der Hauptbibliothek in der Altstadt nur einer kleinerer Handapparat vorgehalten. Der Bestand für die Rechtswissenschaften als nutzungsintensivstes Fach wurde anlässlich der Eröffnung des Triplex-Lesesaals im Sommer 2015 ausgeweitet. Inhaltlich liegen die Schwerpunkte auf Referenzwerken, Präsenzexemplaren von Lehrbüchern sowie umfangreichen Quellenwerken. Ergänzt wird der Buchbestand durch 560 Stellmeter für 110000 Zeitschriftenbände, die - mit Ausnahme der hochfrequentierten Rechtswissenschaften - 14 Tage ausgeliehen werden können.

Entsprechend der Vielfalt der Lern- und Arbeitsanforderungen verfügt der neue Triplex-Lesesaal über ein breites Angebot unterschiedlicher Sitz- und Steharbeitsplätze. Auf den Hauptbewegungsflächen im Zugangsbereich bieten lang gezogene Highboards zwischen den Regalanlagen die Möglichkeit des kurzen Anlesens bzw. der Ablage für die auszuwählende Literatur. Hier befinden sich auch die Recherche-PCs sowie die Infoterminals mit Touchfunktion zur Erstorientierung. An diese schließen die Loungebereiche zum bequemen Lesen an, wobei sich die Lesesessel flexibel um fest installierte kleine Würfel gruppieren, die als Ablagetische und Ladestationen für elektronische Endgeräte dienen. Im Mitteltrakt stehen ferner zwölf Stehpulte zur Verfügung. Das Gros der Arbeitsplätze entfällt auf wissenschaftliche Sitzarbeitsplätze. Unterstützt wird die Form der klassischen Stillarbeit durch das durchgängige Verbot von Jacken, Taschen sowie Lebensmitteln (außer Wasser) und das strikte Verbot, im Lesesaal zu telefonieren.

Im Ostflügel der beiden Hauptgeschosse stehen auf zwei Geschossen zusammen 18 Einzelarbeitskabinen (Carrels) zur Verfügung, die von Doktoranden und Studierenden für eine Abschlussarbeit exklusiv für zwei Monate gemietet werden können. Die Einzelarbeitskabinen sind Sonderanfertigungen. Sie entsprechen einem kleinen Büro mit Tisch, Stuhl, Leuchte, Bücherregalen und weiteren Ablageflächen. Eine Glasschiebetür sorgt für Einsehbarkeit und erhält die Sichtachse zur Grabengasse. Die Lage an der Außenseite der Ostspange bietet den Blick auf den Universitätsplatz.

Zusätzlich zu den klassischen Sitzarbeitsplätzen stehen insgesamt 15 Gruppenarbeitsräume für jeweils vier bis acht Nutzer zum gemeinsamen Lernen und Arbeiten zur Verfügung. Die Gruppenarbeitsräume können über ein Online-Reservierungssystem bis zu zwei Wochen im Voraus reserviert werden. Nach dem Login mit der studentischen Universitätsidentitätsnummer „Uni-ID“, können die Reservierungen jederzeit geändert oder storniert werden. Die Gruppenarbeitsräume sind frei zugänglich 


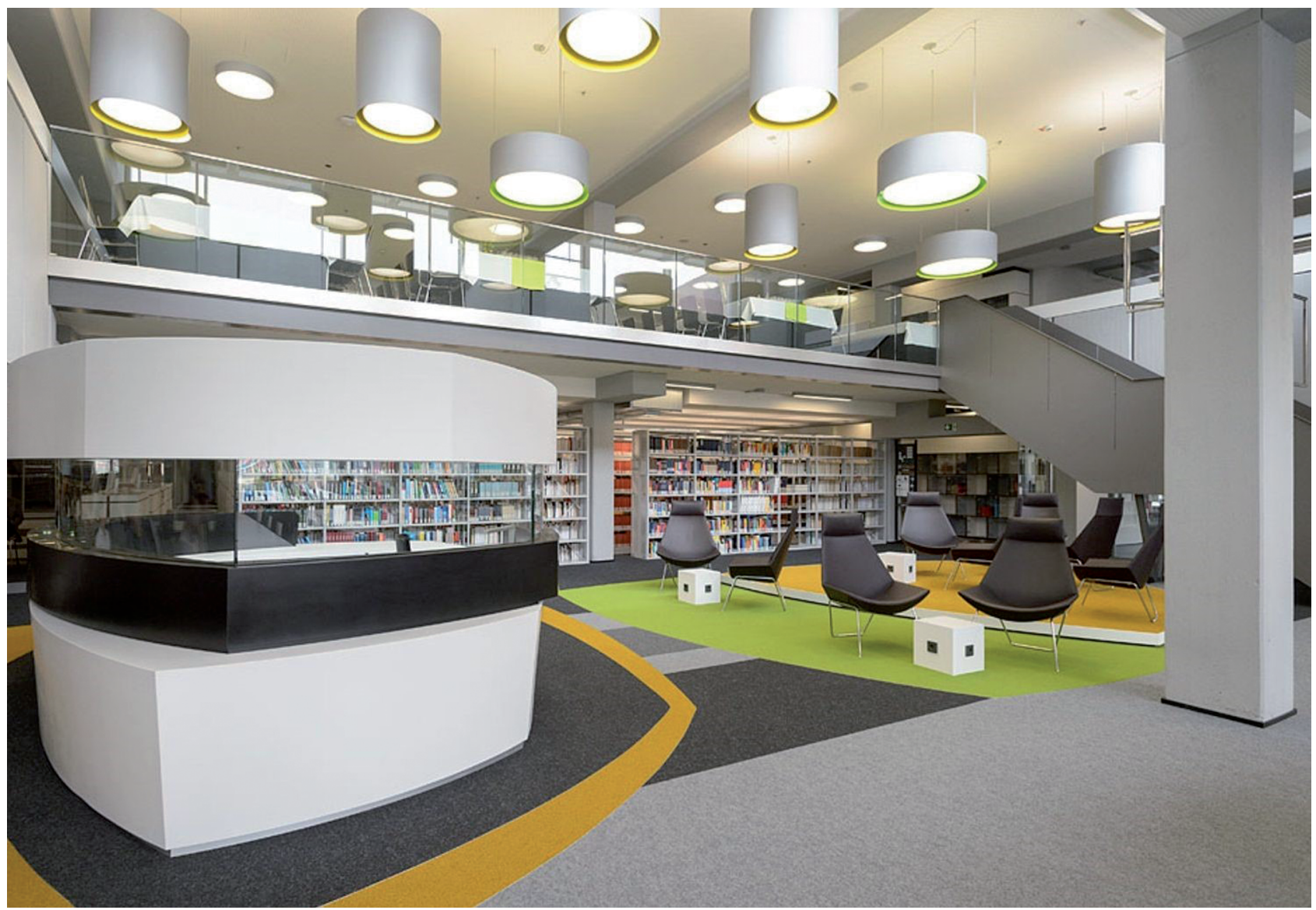

Abb. 6: Informationstheke und Loungebereich im 2. OG. Foto: Philipp Rothe

und können - wenn keine Reservierung vorliegt - auch spontan genutzt werden. Insgesamt vier Infobildschirme zeigen standortspezifisch den aktuellen Reservierungsstand an. Vier Räume verfügen über eine Smartboardbzw. Smartscreen-Lösung. In fünf Räumen wurde das von der Universitätsbibliothek Mannheim entwickelte PalMASystem zum interaktiven Lernen installiert. Dank des „Programms zur Verbesserung der Lehrausstattung an den Hochschulbibliotheken des Landes Baden-Württemberg (PVL-HB BW)“ steht darüber hinaus eine Auswahl an Notebooks und Tablet-Computern mit wissenschaftlichen Applikationen zur Verfügung, die den Einsatz digitaler Arbeitstechniken befördern.

Weitere Angebote im neuen Lesesaal sind eine umfangreiche Auslage von aktuellen Zeitschriftenheften sowie Wochen- und Tageszeitungen, drei Scan- und Kopierräume, ein Eltern-Kind-Raum sowie ein Sonderarbeitsbereich zur Nutzung schützenswerter Bestände. Für das Einschließen von Arbeitsmaterialien stehen im Lesesaal 150 Dauerschließfächer zur Verfügung. Das durchgängige Jacken und Taschenverbot in den Lesesälen wird durch die voranschreitende Umstellung der gesamten Garderoben- anlage der Universitätsbibliothek auf ein Kartensystem unterstützt, das ebenfalls mit der Uni-ID genutzt werden kann.

Sämtliche Umbaumaßnahmen wurden im laufenden Betrieb durchgeführt. Das Ziel, Sperrungen von Arbeitsbereichen sowie Schließungen des gesamten Bibliotheksbetriebs zu vermeiden, konnte bis auf wenige Ausnahmen erreicht werden.

\section{Innovationsfelder an der Heidelberger Universitäts- bibliothek}

Wie eingangs erwähnt, dient in Heidelberg die eigene Tradition als Leitfaden für die Zukunft. Die Bibliotheksleitung plant die Entwicklungsschwerpunkte als Schritte eines organischen Wandlungsprozesses, in den die Bibliothek im vergangenen Jahrzehnt eingetreten ist. Die Norderweiterung in den Triplex fügt sich in eine stete Planung ein, zu der neben der baulichen Erneuerung die Integration des 

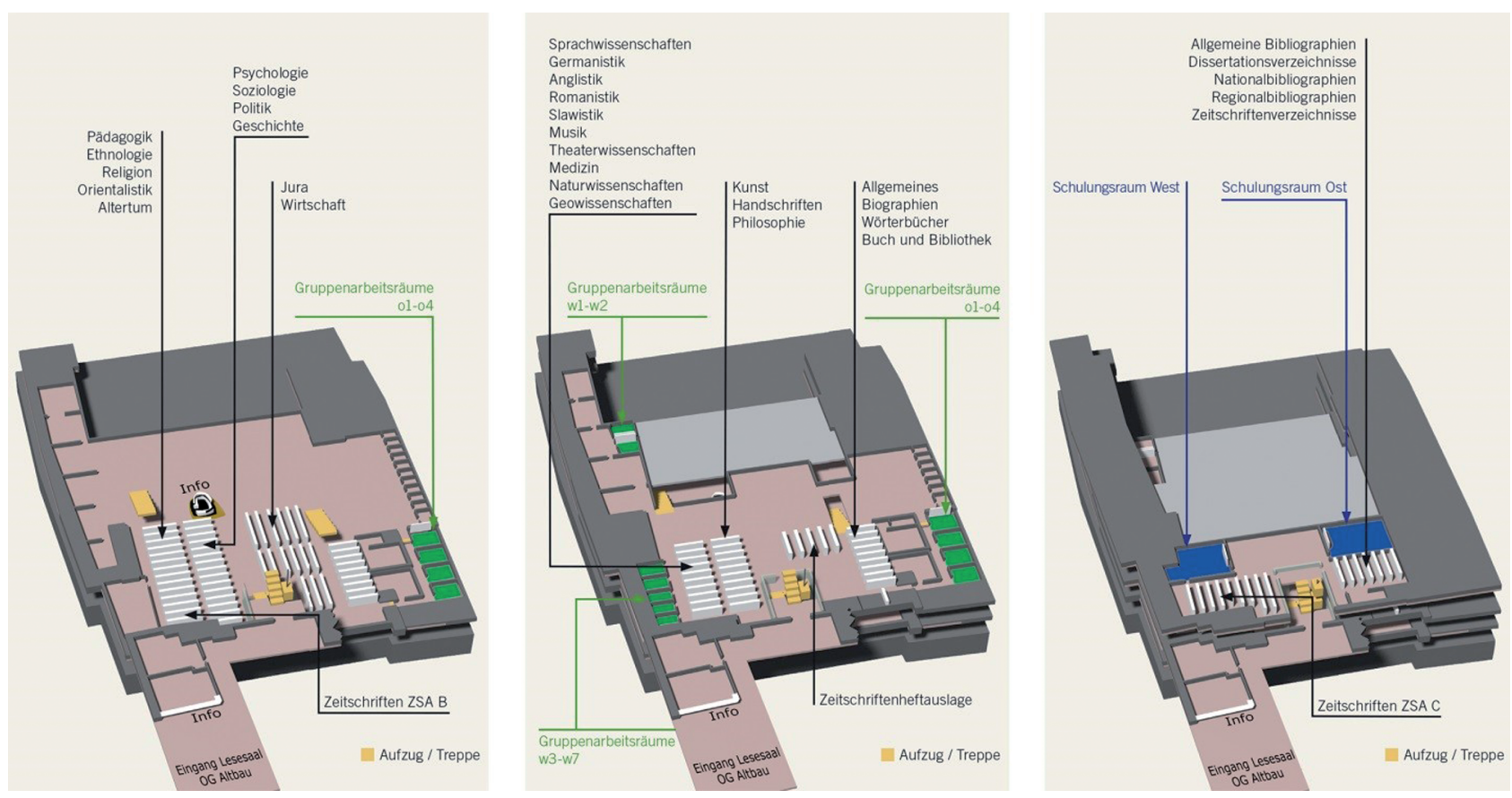

Abb. 7: Lesesaal Triplex: 2.-4. Obergeschoss

Heidelberger Bibliothekssystems, der Ausbau der E-Bibliothek auf der Grundlage innovativer Lizenzverträge sowie der Aufbau hybrider Publikationsdienste gehören. Diese vier Innovationsfelder sollen abschließend aufgezeigt werden.

\section{Bauliche Erneuerung der Universitätsbibliothek}

Mit der Sanierung des Triplex-Gebäudes wurde der zentrale Lernort der Universität Heidelberg in der Altstadt langfristig gestärkt. Dies fügt sich in die Strategie eines betriebswirtschaftlich orientierten Ressourcenmanagements. Mit vergleichsweise geringem Personaleinsatz lassen sich auch in den Randzeiten am Abend sowie an den Wochenenden große Nutzungsflächen betreuen. In den dezentralen Bibliotheken sinkt der Nutzungsdruck.

Aus dieser Überlegung heraus ergeben sich Vorplanungen für die Sanierung weiterer Bereiche des historischen Altbaus. Insbesondere der nördliche Teil des Südflügels mit dem Informationszentrum und dem Multimediazentrum befindet sich auf dem Ausstattungsstand der 1980er-Jahre und bedarf dringend der Sanierung. Ziel ist es, den Altbau dem vorbeugenden Brandschutz entsprechend zu ertüchtigen und zugleich die Aufenthaltsqualität auf den Hauptnutzungsflächen grundlegend zu verbessern. Nach jetzigem Stand ist der Beginn dieser Baumaßnahme für Frühjahr 2017 geplant.

Der historische Magazintrakt als Bindeglied zwischen dem repräsentativen Südflügel und dem funktionalen Triplex-Gebäude wird derzeit ebenfalls Flügel für Flügel brandschutztechnisch ertüchtigt und dadurch in die Lage versetzt, weitere Bestände in Freihandaufstellung aufzunehmen. Durch den Auszug des Lesesaals aus den beiden oberen Geschossen des Magazintraktes besteht die Möglichkeit, weitere Jahrgänge aus dem geschlossenen Tiefenmagazin zu holen und zur sofortigen Ausleihe anzubieten. Von den 3,14 Millionen Bänden des Gesamtbestands werden zukünftig in der Hauptbibliothek 600000 verfügbar sein. Diese Umzugsmaßnahme wird ferner dazu genutzt, die Abfolge der Bestände wieder in eine fortlaufende numerische Signaturenabfolge zu bringen. Ergänzt wird dieser Meilenstein im Bestandsmanagement durch die Überführung weiterer Bereiche des wertvollen historischen Altbestandes in das Tiefenmagazin, das über die erforderlichen klimatischen Bedingungen verfügt.

\section{Integration des Bibliothekssystems}

Gemäß der Verwaltungsordnung von 2004 ist die Universitätsbibliothek Heidelberg beauftragt, die ,ausgeprägte 
Zersplitterung der universitären Bibliothekslandschaft“ $\mathrm{zu}$ überwinden und hinsichtlich des Bestandsmanagements sowie der Nutzungsbedingungen die funktionale Einschichtigkeit herbeizuführen. ${ }^{6}$ Binnen zehn Jahren ist es der Leitung gelungen, die ehemals 104 Bibliotheken (2002) um fast $55 \%$ auf 57 Standorte zu reduzieren. 35 dieser 57 Standorte sind in acht Verwaltungsverbünden zusammengefasst. Die Geschäftsabläufe wurden unter Einführung ITgestützter Prozesse vereinheitlicht und professionalisiert. Ferner setzt der Rückgang des Print-Erwerbungsgeschäftes um 20000 Bände seit 2009 im dezentralen Bibliothekssystem weitere Ressourcen frei, die insbesondere für den Aufbau der Heidelberger Universitätsbibliografie heiBIB genutzt wurden. Mit rund 62000 Publikationseinträgen in vier Jahren zählt heiBIB quantitativ bereits jetzt zu den größeren Universitätsbibliografien im deutschsprachigen Raum.

Die organisatorische Integration neuer Aufgaben wird im dezentralen Bibliotheksbereich durch weitere Baumaßnahmen flankiert, die darauf zielen, ehemals verstreute, fachlich zusammenhängende Einrichtungen räumlich zu verbinden und größere Nutzungsflächen mit verbesserter Ausstattung zu schaffen. Abgeschlossen ist in diesem $\mathrm{Zu}$ sammenhang die oben erwähnte Zusammenführung der Institutsbibliotheken der Wirtschafts- und Sozialwissenschaften in der Campusbibliothek Bergheim im Jahr 2009. ${ }^{7}$ 2012 führte die Universitätsbibliothek die vier Bibliotheken der Theologischen Fakultät im Gebäude des Wissenschaftlich-Theologischen Seminars zusammen. Ferner sind zwei Neubauprojekte geplant bzw. stehen kurz vor der Eröffnung. Anfang 2016 wurde das von der Klaus Tschira Stiftung gestiftete Mathematikon im Neuenheimer Feld bezogen, in das die Bibliotheken der Fakultät für Mathematik und Informatik sowie des Interdisziplinären Zentrums für Wissenschaftliches Rechnen integriert werden. 2015 war der Baubeginn für das Centre for Asian and Transcultural Studies (CATS), das auf dem Campus Bergheim entsteht. Im Zentrum von CATS steht ein Bibliotheksneubau, der vier Stockwerke in die Tiefe gebaut wird. Hier werden fünf Bibliotheken des Südasien-Instituts, des Zentrums für Ostasienwissenschaften und des Instituts für Ethnologie zusammengeführt.

\section{Ausbau der E-Bibliothek}

Die Bereitstellung gedruckter und elektronischer Ressourcen - das bibliothekarische Kerngeschäft der Universitätsbibliothek Heidelberg - betrifft ebenfalls das Verhältnis von Hauptbibliothek und dezentralem Bibliothekssystem. Auch wenn der Literaturetat für gedruckte Ressourcen mit 2,1 Millionen Euro (Referenzjahr 2014) im deutschlandweiten Vergleich noch immer sehr hoch ist, verschiebt sich auch in Heidelberg das Verhältnis zwischen gedruckten und digitalen Ressourcen in Richtung digitaler Angebote. Während der Anteil 2010 noch bei $52 \%$ lag, erreichte er im Referenzjahr 2014 mit 4,2 Millionen Euro bereits 67 \% an den Gesamtausgaben.

Diese Konzentration auf die digitalen Ressourcen erfordert die Lizenzierung der Angebote für das gesamte Heidelberger Bibliothekssystem aus einer Hand. Wegweisend war hier der Vertragsabschluss mit dem SpringerVerlag für das Konsortium Baden-Württemberg im Oktober 2014, in dem unter maßgeblicher Beteiligung Heidelbergs zum ersten Mal ein bestandsunabhängiges E-only-Lizenzmodell durchgesetzt werden konnte. Nicht nur die Titel des Springer-Verlags, sondern auch die Zeitschriften von Elsevier, Wiley-Blackwell, Sage sowie Cambridge und Oxford University Press werden durch eine neue Form der Gemeinschaftsfinanzierung in Heidelberg bereitgestellt, weitere Verlage werden folgen. Die bisher von den Heidelberger Instituten dezentral verausgabten Mittel für Einzelabonnements werden dazu an der Universitätsbibliothek zusammengeführt und von hier aus in Konsortial- und DFG-Allianzverträge eingebracht, die der Universität den dauerhaften Onlinezugriff auf die Komplettpakete der Verlage sichern. Diese Entwicklung geht nicht nur mit einer starken Verwaltungsvereinfachung einher, sondern bereichert die Informationsversorgung auch qualitativ. So wurde beispielsweise das Portfolio des Springer-Verlages in Heidelberg von 660 auf 1900 Titel erweitert. Die fortschreitende Zentralisierung der E-Medien-Verwaltung erzielt Synergieeffekte. Diese führen zu einer weiteren Entlastung der dezentral eingesetzten personellen Ressourcen, die umgewidmet und für den Ausbau der neuen Entwicklungsfelder der Bibliothek eingesetzt werden können.

\section{Publikationen, Forschungsdaten, Digitale Editionen}

Neben der baulichen Erneuerung, der Integration des Bibliothekssystems und dem zentralen Lizenzmanagement sind die Publikationsdienste ein weiteres zentrales Inno-
6 Verwaltungsordnung für das Bibliothekssystem der Universität Heidelberg (23.03.2004) unter http://www.ub.uni-heidelberg.de/all g/profil/jurbasics/Verwaltungsordnung_2003.pdf.

7 Vgl. ausführlich Krüger (2014). 
vationsfeld. Die Bibliothek verfolgt hier das Ziel, sich langfristig vom reinen Informationsvermittler zum Informationsproduzenten weiter zu entwickeln. Der Startschuss für diese Entwicklung fiel mit dem Aufbau der Digitalisierungswerkstatt im Jahr 2002, als die Universitätsbibliothek einen Grazer Buchtisch erwarb und mit der Digitalisierung spätmittelalterlicher Bilderhandschriften die ersten digitalen Daten produzierte. Als weitere Meilensteine sind die systematische Digitalisierung der deutschsprachigen, seit 2012 auch der lateinischen und griechischen Handschriften der Bibliotheca Palatina $\mathrm{zu}$ nennen, die virtuelle Zusammenführung der karolingischen Klosterbibliothek Lorsch in der Bibliotheca Laureshamensis, die Digitalisierung von Auktionskatalogen der Jahre 1901 bis 1945 zur Klärung der Provenienz von Kunstwerken, insbesondere bei Raubkunstverdacht, sowie die Massendigitalisierung im Rahmen verschiedener DFG-Projekte. Die Entwicklung eines Annotationsmoduls für Wissenschaftler zur Kommentierung von Texten und Bildern als Erweiterung der an der Universitätsbibliothek entwickelten Digitalisierungssoftware DWork, das auch in andere Softwarelösungen eingebunden werden kann, ist das neueste Aktionsfeld in diesem Bereich.

Einen weiteren Schwerpunkt bilden die digitalen Editionen bedeutender Textkorpora. So kooperiert die Universitätsbibliothek mit dem Heidelberger Sonderforschungsbereich „Materiale Textkulturen“. ${ }^{8}$ Als erstes Projekt wurde der "Welsche Gast", eine mittelhochdeutsche Verhaltenslehre von Thomasin de Zerklaere aus dem Jahr 1215 editiert, die in 25 Handschriften und Fragmenten überliefert ist. Die digitale Präsentation stellt die Textzeugen als Digitalisate mit Transkription und Übersetzung am Bildschirm nebeneinander angeordnet zum Vergleich bereit. ${ }^{9}$ Mit der Kaiserchronik, einer um die Mitte des 12. Jahrhunderts entstandenen, frühmittelhochdeutschen Reimchronik, entsteht eine weitere Digitale Edition in Zusammenarbeit mit der University of Cambridge. ${ }^{10}$

Digitalisierung und Digitale Editionen sind Teil einer weiter gefassten Open-Access-Strategie, bei der die Universitätsbibliothek Heidelberg für die Publikation digitaler Inhalte mit verschiedenen nationalen und internationalen Partnern kooperiert. Neben etablierten Diensten wie der Bild- und Multimediadatenbank heidICON, die rund

8 SFB 933, Teilprojekt B06: Materiale Präsenz des Geschriebenen und ikonographische Rezeptionspraxis in der mittelalterlichen Lehrdichtung. Text-Bild-Edition und Kommentar zum Welschen Gast des Thomasin von Zerklaere. Verfügbar unter http://www.materiale-text kulturen.de/teilprojekt.php?tp=B06\&up.

9 http://digi.ub.uni-heidelberg.de/wgd.

10 http://www.mml.cam.ac.uk/german/research/kaiserchronik.
400000 Objekte über die Heidelberger Instanz sowie aktuell rund 100000 Objekte über die Deutsche Digitale Bibliothek verfügbar macht, wie auch die Publikationsserver heiDOK, ART-Dok, Propylaeum-DOK und das CrossAsiaRepository mit insgesamt rund 27500 Volltexten werden derzeit weitere Angebote aufgebaut. Die Publikationsdienste stehen auch bei den drei Fachinformationsdiensten (FID) im Zentrum, die die Universitätsbibliothek Heidelberg zusammen mit der Sächsischen Landesbibliothek Staats- und Universitätsbibliothek Dresden (Kunst, Fotografie, Design), der Bayerischen Staatsbibliothek München (Altertumswissenschaften) und der Staatsbibliothek zu Berlin (Asienwissenschaften) betreibt. Ein zentrales Ziel beim Ausbau der FIDs besteht darin, die jeweiligen Fachcommunitys durch das Angebot digitaler Publikationsmöglichkeiten in ihrer wissenschaftlichen Arbeit zu unterstützen.

In Auftrag der Universität koordiniert die Bibliothek die campusweiten Open-Access-Aktivitäten und baut mit dem Universitätsrechenzentrum ein gemeinsames Kompetenzzentrum Forschungsdaten (KFD) mit dem Dienst heiDATA für das Forschungsdatenmanagement auf. ${ }^{11}$ In heiDATA, das die an der Harvard University entwickelte Open-Source-Software Dataverse einsetzt, sind aktuell 55 Studien in 14 Dataverses aus 10 übergeordneten Einrichtungen der Universität abgelegt. Im Rahmen der E-ScienceFörderlinie des Landes Baden-Württemberg baut das KFD einen Pool mit generischen Softwarewerkzeugen zur Erstellung dynamischer Publikationsportale für Forschungsdaten auf, ein dringendes Desiderat der Wissenschaftler zur Präsentation ihrer Daten. Ein weiteres Ziel des Zentrums besteht darin, für große Datenmengen Konzepte zur langfristigen Archivierung zu entwickeln und den Umgang mit Forschungsdaten als eigenständige Thematik in der universitären Lehre und Forschung zu verankern.

An der Spitze der Heidelberger Neuentwicklungen steht der Verlag Heidelberg University Publishing (heiUP), den die Universität 2015 gegründet hat. Aufbauend auf einer DFG-Förderung, die die Bibliothek gemeinsam mit dem Heidelberger Exzellenzcluster Asien und Europa eingeworben hat, entsteht eine komplexe Infrastruktur zur digitalen Publikation herausragender Forschungsergebnisse im Open Access. Die Qualität der Verlagsproduktion Monografien, Serien und Zeitschriften - wird durch ein doppeltes Peer-Review-Verfahren sichergestellt, das von einem wissenschaftlichen Beirat eingeleitet wird. Eine konsequente E-Strategie, die Nutzung innovativer digitaler $\mathrm{Pu}$ blikationsformate sowie die Herstellung hochwertiger

$11 \mathrm{http} / / /$ data.uni-heidelberg.de. 


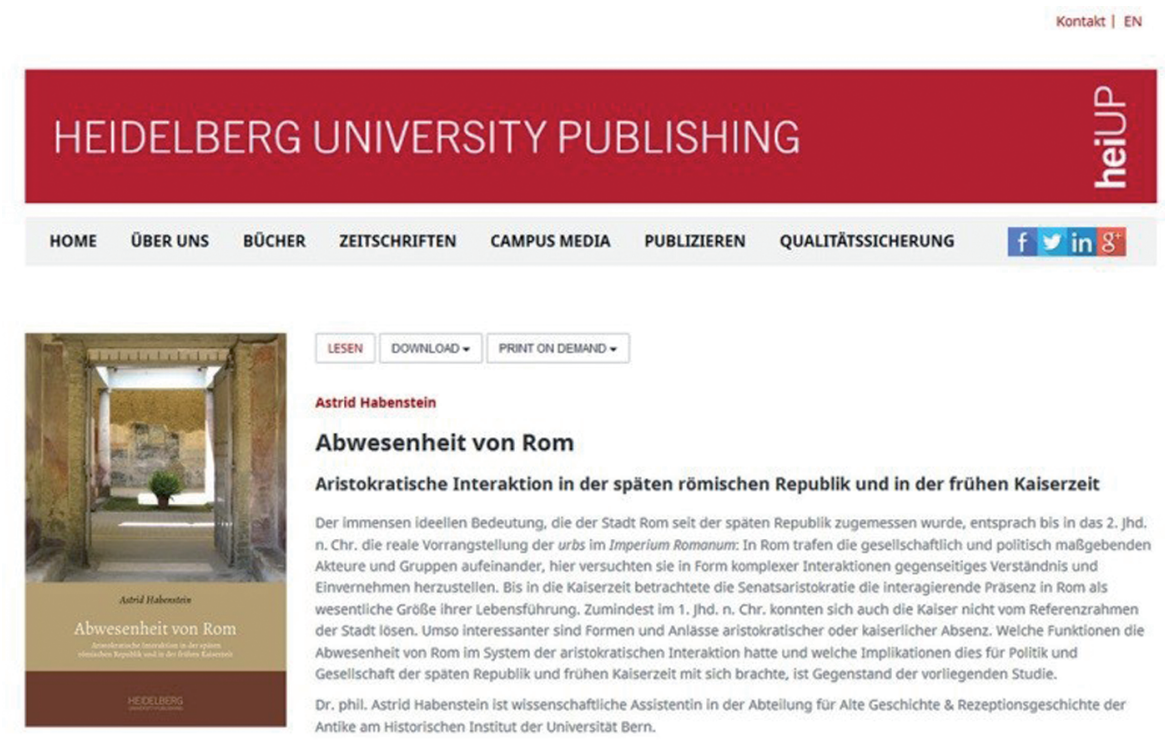

Abb. 8: Verlagswebseite Heidelberg University Publishing

Druckausgaben im Print-on-Demand-Verfahren ergänzen das Angebot. Zum Einsatz kommen hier die international verbreiteten Open-Source-Lösungen Open Journal Systems (OJS) und Open Monograph Press (OMP) des Public Knowledge Projects (PKP), die in einer Entwicklergemeinschaft unter Beteiligung Heidelberger IT-Spezialisten der Universitätsbibliothek weiterentwickelt werden.

Die Bereitstellung von gekauften Print- und lizenzierten Online-Medien wird auch künftig zum Dienstleistungsportfolio der Universitätsbibliothek Heidelberg zählen. Doch zunehmend ergänzt wird dieses klassische Portfolio durch den Ausbau digitaler Publikationsangebote, die mit verschiedenen Akteuren aus der Wissenschaft zusammen weiterentwickelt werden. Im Zentrum stehen dabei die von der Bibliothek betriebenen Fachinformationsdienste sowie heiDOK, heidICON, heiDATA und heiUP als zentrale Angebote für die wissenschaftliche Community.

\section{Ausblick}

Durch die Eröffnung des Triplex-Lesesaals im Sommer 2015 wird die Universitätsbibliothek Heidelberg ihrem Anspruch gerecht, ihren Nutzern auch räumlich ein attraktives Angebot zu unterbreiten. Durch die Sanierung dieses unmittelbar nördlich an den Altbau anschließenden Gebäudekomplexes ist eine zusätzliche Nutzungsfläche von 6500 Quadratmetern mit hoher Aufenthaltsqualität entstanden. Die im Vergleich zum Vorjahreszeitraum deutliche Steigerung der Nutzungszahlen um $88 \%$ zeugt von der Attraktivität dieses Angebots. Im Anschluss an diese
Baumaßnahme wird die Sanierung des historischen Altbaus unter Neugestaltung der großen Empfangsbereiche fortgesetzt. Flankiert wird die Verbesserung der Bibliotheksausstattung am Heidelberger Standort durch die $\mathrm{Zu}$ sammenführung weiterer Institutsbibliotheken zu großen Bereichsbibliotheken in Neubauten. Beispielhaft dafür stehen das Mathematikon und das neue Asienzentrum.

Was die Triplex-Norderweiterung für die Zukunftsfähigkeit der Universitätsbibliothek Heidelberg vor Ort bedeutet, das bedeuten die Eigenentwicklungen des Hauses im Bereich digitaler Dienste für ihre nationale und internationale Sichtbarkeit. Dazu zählt die Entwicklung zukunftsweisender Services aus bestehenden Angeboten heraus unter aufmerksamer Beobachtung der konkreten Bedarfe der jeweiligen Zielgruppe. Im Wesentlichen ist dies die klassische Bereitstellung eines reichen Angebots an gedruckten und digitalen Ressourcen, arrondiert um eigenentwickelte Publikationsdienstleistungen.

All diese Neuentwicklungen wären nicht denkbar ohne Förderung aus dritter Hand. So bewirbt sich die Universitätsbibliothek Heidelberg bei verschiedenen Forschungsförderungsinstitutionen um Unterstützung. Die Deutsche Forschungsgemeinschaft, das Ministerium für Wissenschaft, Forschung und Kunst Baden-Württemberg und die Manfred-Lautenschläger-Stiftung sind als langjährige Förderer besonders hervorzuheben. Zuletzt warb die Universitätsbibliothek im Jahr 2015 insgesamt über zwei Millionen Euro an Drittmitteln ein. Gefördert werden derzeit drei Fachinformationsdienste für die Wissenschaft, drei Projekte im Bereich Open-Access-Publikationen und Forschungsdaten, fünf Digitalisierungsprojekte und zwei 
Projekte zur Handschriften- und Nachlasserschließung. ${ }^{12}$ Diese 13 laufenden Drittmittelprojekte zeugen davon, dass die Universitätsbibliothek Heidelberg die Wandlung vom reinen Informationsvermittler zum Informationserzeuger vollzieht und sich damit, im siebten Jahrhundert ihrer Geschichte, neu erfindet.

\section{Literaturverzeichnis}

Altstadterneuerung (1970): Altstadterneuerung in Heidelberg. In: Bauwelt, 61 (32), 1226-37.

Grammbitter-Ostermann, Ulrike (1985): Die Universitätsbibliothek. In: Semper apertus: Sechshundert Jahre Ruprecht-Karls-Universität Heidelberg, 1386-1986: Festschrift in sechs Bänden, hg. v. Wilhelm Doerr, 184-202. Berlin u. a.: Springer.

Kranz, Gabriele (1985): Das Seminargebäude Grabengasse/Sandgasse. In: Semper apertus: Sechshundert Jahre Ruprecht-Karls-Universität Heidelberg, 1386-1986: Festschrift in sechs Bänden, hg. v. Wilhelm Doerr, 203-10. Berlin u. a.: Springer.

Krüger, Marion (2014): Die Campus-Bibliothek Bergheim an der Universität Heidelberg. Das Herz der Fakultät schlägt in der ehemaligen Kardiologie. In: Handbuch Hochschulbibliothekssysteme: Leistungsfähige Informationsinfrastrukturen für Wissenschaft und Studium, hg. v. Konstanze Söllner und Wilfried Sühl-Strohmenger, 524-35. Berlin: De Gruyter Saur.

12 http://www.ub.uni-heidelberg.de/wir/projekte.html.

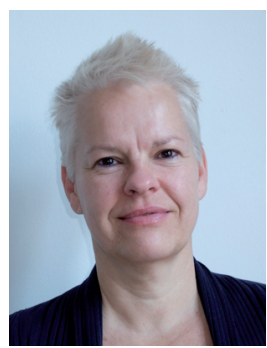

Rike Balzuweit

Universitätsbibliothek Heidelberg

Plöck 107-109

D-69117 Heidelberg

Balzuweit@ub.uni-heidelberg.de

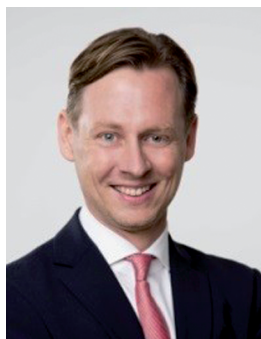

\section{Dr. Martin Nissen}

Universitätsbibliothek Heidelberg

Plöck 107-109

D-69117 Heidelberg

Nissen@ub.uni-heidelberg.de 